\title{
MCA1 and MCA2 Are Involved in the Response to Hypergravity in Arabidopsis Hypocotyls
}

\author{
Takayuki Hattori ${ }^{1, \dagger}$, Yasuhiro Otomi ${ }^{1, \dagger}$, Yohei Nakajima ${ }^{1}$, Kouichi Soga ${ }^{1}(\mathbb{D}$, \\ Kazuyuki Wakabayashi ${ }^{1}$, Hidetoshi Iida ${ }^{2}$ and Takayuki Hoson ${ }^{1, * \mathbb{D}}$ \\ 1 Department of Biology, Graduate School of Science, Osaka City University, Sumiyoshi-ku, Osaka 558-8585, \\ Japan; d20sc005@dt.osaka-cu.ac.jp (T.H.); o_mix_episode2@msn.com (Y.O.); \\ d4c.ballbreaker.29@gmail.com (Y.N.); soga@sci.osaka-cu.ac.jp (K.S.); wakaba@sci.osaka-cu.ac.jp (K.W.) \\ 2 Department of Biology, Tokyo Gakugei University, Koganei, Tokyo 184-8501, Japan; iida@u-gakugei.ac.jp \\ * Correspondence: hoson@sci.osaka-cu.ac.jp \\ + These authors contributed equally to this study.
}

Received: 21 March 2020; Accepted: 3 May 2020; Published: 5 May 2020

\begin{abstract}
Plants respond to and resist gravitational acceleration, but the mechanism of signal perception in the response is unknown. We studied the role of MCA (mid1-complementing activity) proteins in gravity perception by analyzing the expression of the MCA1 and MCA2 genes, and the growth of hypocotyls of mca mutants, under hypergravity conditions in the dark. An MCA1 promoter::GUS fusion reporter gene construct (MCA1p::GUS) and MCA2p::GUS were expressed almost universally in etiolated seedlings. Under hypergravity conditions, the expression levels of both genes increased compared with that under the $1 g$ condition, and remained higher, especially in the basal supporting region. On the other hand, mca-null and MCA-overexpressing seedlings showed normal growth under the $1 \mathrm{~g}$ condition. Hypergravity suppressed elongation growth of hypocotyls, but this effect was reduced in hypocotyls of mca-null mutants compared with the wild type. In contrast, $M C A$-overexpressing seedlings were hypersensitive to increased gravity; suppression of elongation growth was detected at a lower gravity level than that in the wild type. These results suggest that MCAs are involved in the perception of gravity signals in plants, and may be responsible for resistance to hypergravity.
\end{abstract}

Keywords: Arabidopsis; gravity resistance; hypergravity; MCA1; MCA2; mechanosensitive channel; signal perception

\section{Introduction}

Plants are exposed to a variety of mechanical stresses such as wind and gravity, and they have developed efficient mechanisms to respond to these stressors over evolutionary time, in particular after their emergence on land. We have previously studied the mechanisms of plant responses to gravitational acceleration, mainly with basipetal hypergravity produced by centrifugation $[1,2]$. Hypergravity generally suppresses elongation growth and increases the rigidity of plant organs. The cell wall is an important source of mechanical strength in plants; it is likely that the properties of the cell wall are also modified under hypergravity conditions. Hypergravity induces changes in cell wall metabolism, such as accumulation of its constituents, polymerization of certain matrix polysaccharides due to breakdown suppression, stimulation of cross-link formation, and modifications to the wall environment, which lead to increased cell wall rigidity [3]. Hypergravity promotes the lateral expansion of plant organs, with concomitant suppression of elongation growth [4]. Cortical microtubules are likely responsible for the anisotropic growth of plant cells $[5,6]$. Hypergravity induces reorientation of 
cortical microtubules from transverse to longitudinal directions [4], and increases the expression of tubulin and microtubule-related genes [7-9], which may be responsible for the reorientation.

The mechanism of gravity signal perception in the response of plants to hypergravity has been examined. Gadolinium ions, blockers of mechanosensitive ion channels $[10,11]$, nullified the hypergravity-induced modifications to growth anisotropy and cell wall rigidity [12,13]. Hypergravity-induced upregulation of the tubulin- and microtubule-related genes was also suppressed by gadolinium ions [8,9]. Moreover, the gadolinium ions decreased the alignment angle of the epidermal cell files and the cortical microtubules of tubulin mutants at $1 g$, and hypergravity did not affect these factors when the gadolinium ions were present [14]. These results suggest that mechanosensitive ion channels are involved in the plant's response to hypergravity, and have contributed to the concept of 'gravity resistance' [1,2]. However, the mechanosensitive ion channels have yet to be identified at the molecular level.

Candidates for the mechanosensitive ion channels have been reported in Arabidopsis; MCA (mid1-complementing activity) proteins are one such candidate. The amino acid sequences of the MCA1 (At4g35920) and MCA2 (At2g17780) proteins share 73\% homology and partially complement the yeast mid1 mutant, which lacks a putative $\mathrm{Ca}^{2+}$-permeable mechanosensitive channel $[15,16]$. Both proteins are localized in the plasma membrane, and have a single transmembrane segment, as well as an EF-hand-like motif and a coiled-coil motif in the $\mathrm{N}$-terminal half and a plac8 motif in the C-terminal half $[15,17]$. Multiple lines of evidence suggest that MCA1 and MCA2 are involved in mechanosensation. MCA1 is required for sensing substrate (agar medium or soil) hardness [15]. MCA1 and MCA2 are responsible for $\mathrm{Ca}^{2+}$ influx in yeast cells and Arabidopsis seedlings $[15,16]$, a hypo-osmotic shock-induced increase in the intracellular $\mathrm{Ca}^{2+}$ concentration $[15,18]$, and membrane stretch-induced cation currents in Xenopus laevis oocytes [19]. Both proteins are also involved in the cold-induced elevation of $\mathrm{Ca}^{2+}$ and cold tolerance [20]. In the present study, we examined the role of MCA1 and MCA2 in gravity perception by analyzing the expression of the MCA1 and MCA2 genes, and the growth of etiolated hypocotyls of mca mutants, under hypergravity conditions.

\section{Results}

\subsection{Hypergravity-induced Expression of MCA Genes}

The expression patterns of $M C A 1$ and $M C A 2$ in etiolated seedlings grown at $300 \mathrm{~g}$ were analyzed using an MCA1 promoter::GUS fusion reporter gene construct (MCA1p::GUS) and MCA2p::GUS. MCA1p::GUS was expressed in the cotyledons and hypocotyls, regardless of age or gravitational conditions (Figure 1). In the hypocotyls, MCA1p::GUS was strongly expressed in the vascular bundles. Under hypergravity conditions, the expression of MCA1 increased in the hypocotyls compared to the $1 \mathrm{~g}$ conditions. MCA1 expression remained higher in the middle and basal regions.

MCA2p::GUS was also expressed in the cotyledons and hypocotyls (Figure 2). In the hypocotyls, MCA2p::GUS was expressed in the vascular bundles and the parenchymal and epidermal cells. The expression levels decreased from the apical to the basal regions of the hypocotyls. Under hypergravity conditions, $M C A 2$ expression increased in all regions of the hypocotyls compared to $1 g$, and remained higher, particularly in the basal region. 


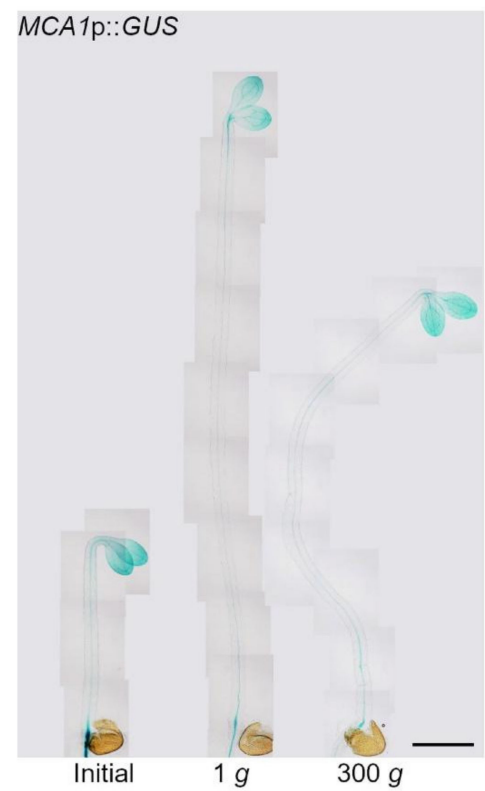

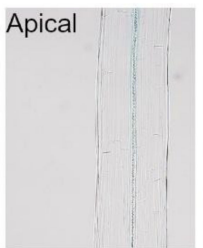
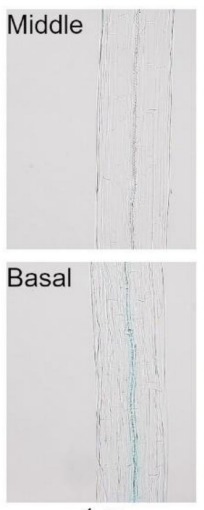

$1 \mathrm{~g}$
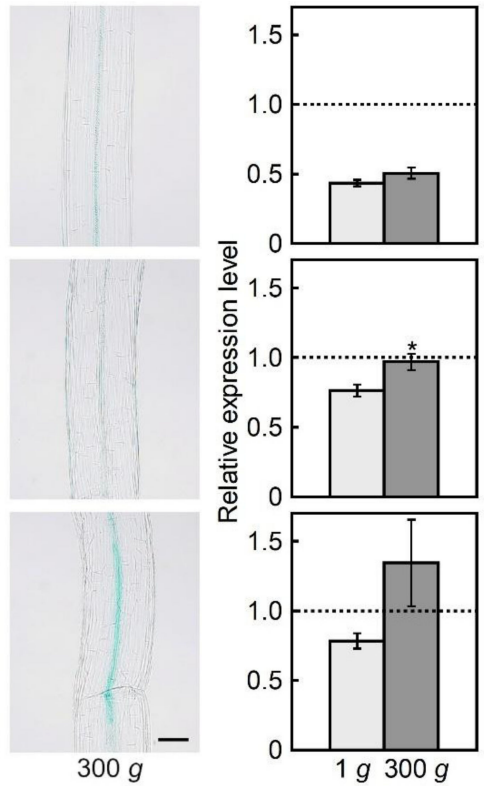

Figure 1. Effects of hypergravity on MCA1 gene expression. An MCA1p::GUS transgenic line was grown at $25^{\circ} \mathrm{C}$ for $48 \mathrm{~h}$ at $1 \mathrm{~g}$. The seedlings were then transferred to $1 \mathrm{~g}$ or $300 \mathrm{~g}$ conditions and grown at $25^{\circ} \mathrm{C}$ for an additional $24 \mathrm{~h}$. Left, GUS-stained seedlings (bar $=1 \mathrm{~mm}$ ). Middle, magnification of the hypocotyls (bar $=0.1 \mathrm{~mm}$ ). Right, quantified GUS-staining intensity (expression level) of each region of the hypocotyl. The expression level was normalized to the initial mean value. The dashed line indicates the initial values. Values are means \pm SE $(n=10-12) .{ }^{*}$ Mean value with a significant difference between the $1 g$ and $300 g$ treatments (Student's $t$-test: $p<0.05$ ).
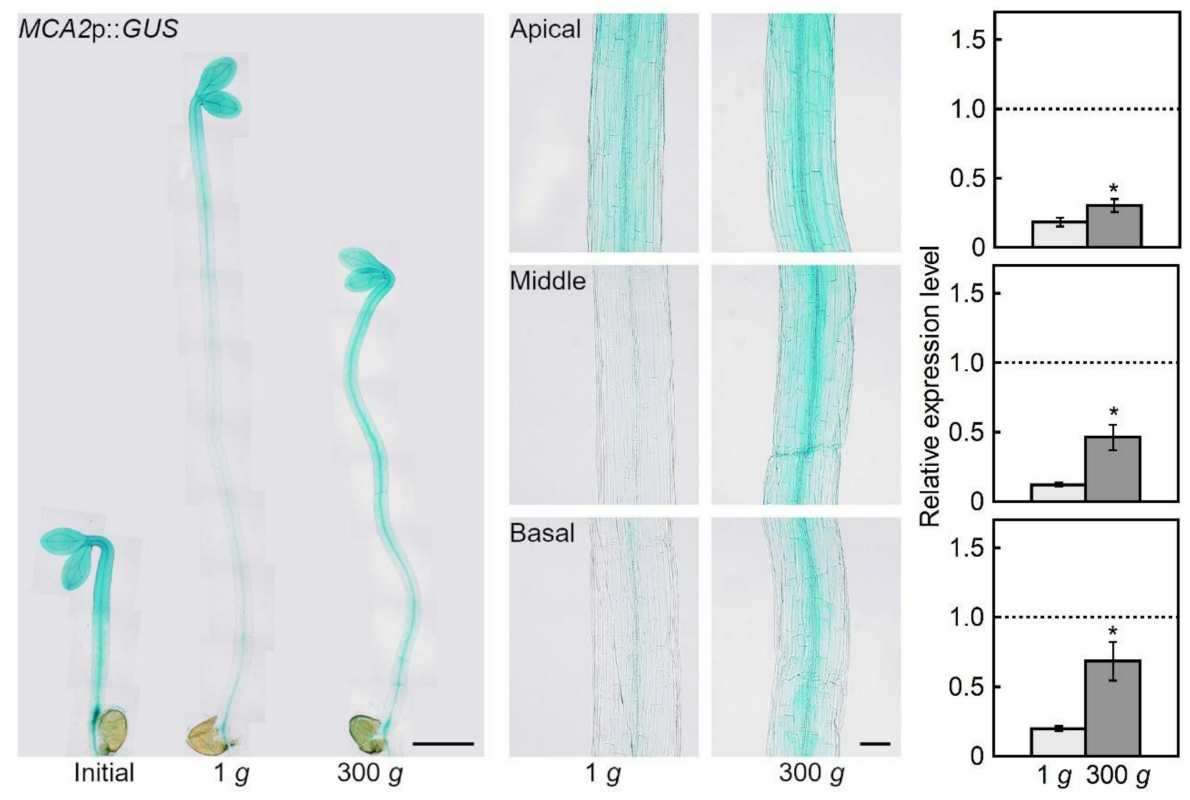

Figure 2. Effects of hypergravity on MCA2 gene expression. An MCA2p::GUS transgenic line was grown as described in Figure 1. Left, GUS-stained seedlings (bar $=1 \mathrm{~mm}$ ). Middle, magnification of the hypocotyls (bar $=0.1 \mathrm{~mm}$ ). Right, quantified GUS-staining intensity (expression level) of each region of the hypocotyl. The expression level was normalized to the initial mean value. The dashed line indicates the initial values. Values are means \pm SE $(n=10-12) .{ }^{*}$ Mean value with a significant difference between the $1 \mathrm{~g}$ and $300 \mathrm{~g}$ treatments (Student's $t$-test: $P<0.05$ ). 


\subsection{Hypergravity-Induced Growth of Mca-Null and MCA-Overexpression Mutants}

The growth of the etiolated hypocotyls of mca-null and MCA-overexpressing mutants under hypergravity conditions was analyzed to assess if MCA1 and MCA2 are involved in gravity perception, in the response to increased gravity. The growth of the mca1-null, mca2-null, and mca1-null mca2-null double mutants (double mutant) was normal in the dark at $1 g$ (Figure 3), although the double mutant showed growth defects in the light [16]. Under hypergravity conditions at $300 g$, elongation growth of the hypocotyls was suppressed by $30 \%$ in the wild type. Hypergravity at $300 \mathrm{~g}$ also suppressed elongation growth of the hypocotyls in the mca1-null, mca2-null, and double mutants. However, the suppression ratio of the three mca-null mutants was smaller than that of the wild type, and was $16 \%-21 \%$ (Figure 3). There were no differences in the growth suppression ratios of the three mca-null mutants. In a higher gravity condition at $500 \mathrm{~g}$, growth suppression was slightly enhanced in the wild type and mca1-null mutant, but not in the mca2-null or double mutants (Figure 3). The level of elongation growth suppression by $500 \mathrm{~g}$ hypergravity was similar between the mca1-null, mca2-null, and double mutants.
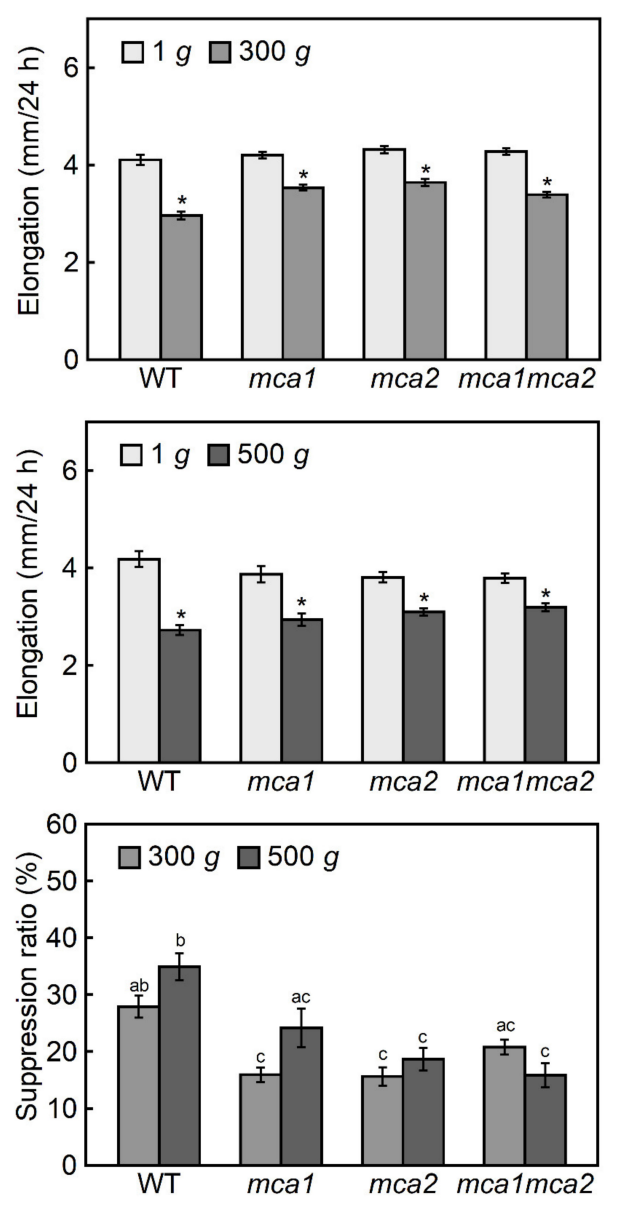

Figure 3. Effects of hypergravity on the elongation growth of hypocotyls in mca-null mutants. Wild type (WT) and mca-null mutants were grown at $25^{\circ} \mathrm{C}$ for $48 \mathrm{~h}$ at $1 \mathrm{~g}$. The seedlings were then transferred to $1 \mathrm{~g}, 300 \mathrm{~g}$, or $500 \mathrm{~g}$ conditions and grown for an additional $24 \mathrm{~h}$ at $25^{\circ} \mathrm{C}$. The length of the hypocotyls was measured using a scale. Values are means \pm SE $(n=20)$. Upper, elongation under $1 g$ or 300 $g$ conditions after $24 \mathrm{~h}$ growth. Middle, elongation under $1 \mathrm{~g}$ or $500 \mathrm{~g}$ conditions after $24 \mathrm{~h}$ growth. * Mean value with a significant difference between the $1 g$ and hypergravity treatments in each line (Student's $t$-test: $p<0.05$ ). Lower, suppression ratio (the difference between the elongation under $1 g$ and hypergravity conditions was shown as a percentage of the $1 g$ value). Different letters above the bars represent statistically significant differences (Tukey's HSD test: $p<0.05$ ). 
Seedling growth of the $M C A 1$-overexpressing and $M C A 2$-overexpressing mutants in the dark at $1 \mathrm{~g}$ was normal (Figure 4), but the MCA1-overexpressing mutant showed growth defects in light [15]. At $300 \mathrm{~g}$, the elongation growth of the hypocotyls was suppressed by $30 \%-35 \%$, and there were no differences in the growth suppression ratios of the wild type or MCA1- and $M C A 2$-overexpressing mutants. Under lower gravity conditions at $30 g$, the wild type exhibited no elongation growth suppression, but a small significant suppression was observed in the MCA1- and the MCA2-overexpressing mutants (Figure 4).
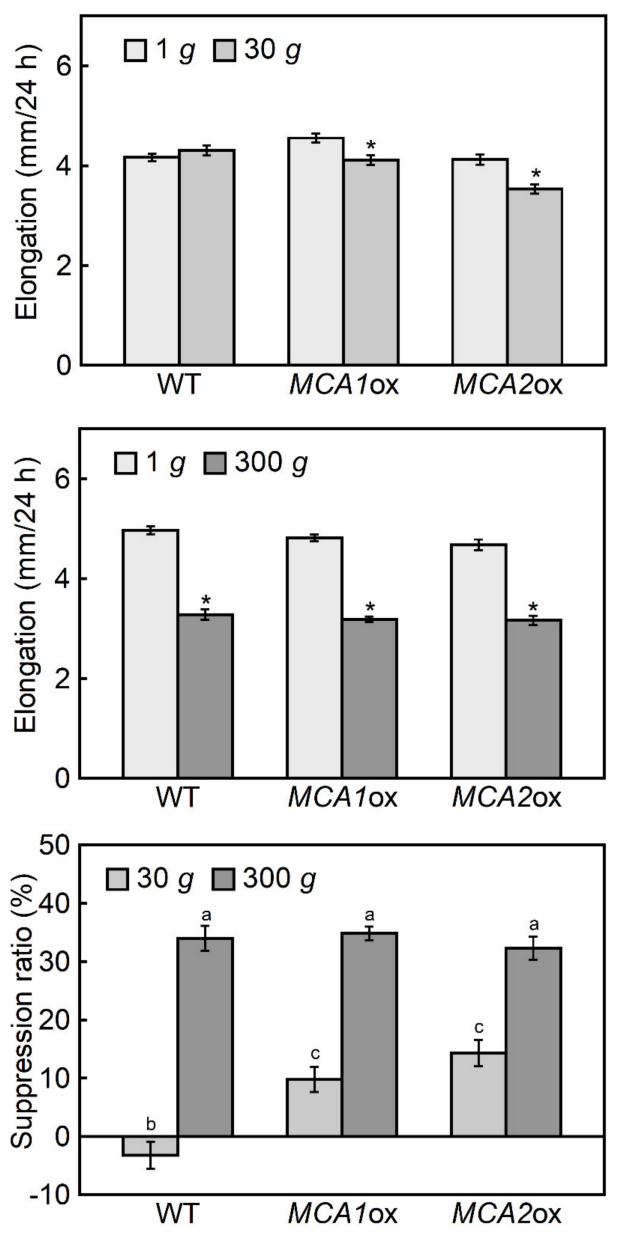

Figure 4. Effects of hypergravity on the elongation growth of hypocotyls in $M C A$-overexpressing mutants. Wild type (WT) and MCA-overexpressing mutants (MCA1ox and MCA2ox) were grown on agar medium at $25^{\circ} \mathrm{C}$ for $48 \mathrm{~h}$ at $1 \mathrm{~g}$. Seedlings were then transferred to $1 \mathrm{~g}, 30 \mathrm{~g}$, or $300 \mathrm{~g}$ conditions and grown for an additional $24 \mathrm{~h}$ at $25^{\circ} \mathrm{C}$. The length of the hypocotyls was measured using a scale. Values are means \pm SE $(n=20)$. Upper, elongation under $1 g$ or $30 g$ conditions after $24 \mathrm{~h}$ growth. Middle, elongation under $1 \mathrm{~g}$ or $300 \mathrm{~g}$ conditions after $24 \mathrm{~h}$ growth. ${ }^{*}$ Mean value with a significant difference between the $1 g$ and hypergravity treatments in each line (Student's $t$-test: $p<0.05$ ). Lower, suppression ratio (the difference between the elongation under $1 g$ and hypergravity conditions was shown as a percentage of the $1 g$ value). Different letters above the bars represent statistically significant differences (Tukey's HSD test: $p<0.05$ ).

\section{Discussion}

In the present study, we used etiolated seedlings to avoid the influence of light-the light response pathway overlaps with the gravity response and acts as a gravity-substitution factor in plant growth regulation [1,21]. MCA1p::GUS and MCA2p::GUS were expressed almost universally in the etiolated seedlings (Figures 1 and 2), which is consistent with observations from light-grown 
seedlings [16]. Under hypergravity conditions, the expression levels of both genes increased compared to $1 \mathrm{~g}$. Preliminary microarray analyses showed that the expression levels of MCA1 and MCA2 were higher under hypergravity conditions, but lower under microgravity conditions in space compared to $1 \mathrm{~g}$. These results indicate that the expression of both genes is regulated by gravitational conditions. The amount of signal receptors affects the sensitivity to a given signal, so plants would likely prepare for gravitational stress by increasing the expression of $M C A 1$ and $M C A 2$ to enhance gravity sensitivity.

The level of GUS expression in the MCA1p::GUS line (Figure 1) was lower than in the MCA2p::GUS line (Figure 2). This result is consistent with the same lines grown under light conditions [16]. However, our observations may not necessarily reflect the actual expression levels of MCA1 and MCA2, because GUS expression often varies from line to line. According to preliminary microarray analyses, the expression level of $M C A 1$ was several times higher than that of $M C A 2$ under $1 g$ conditions.

The growth of the etiolated hypocotyls of $m c a$ mutants was analyzed under hypergravity conditions to investigate the role of MCA1 and MCA2 in gravity perception, in the response to increased gravity. In general, the mca-null and $M C A$-overexpressing seedlings grew normally in the dark at $1 g$ (Figures 3 and 4), although some growth defects were observed in light-grown mature plants $[15,16]$. Under hypergravity conditions, elongation growth was suppressed, but this effect was reduced in the mca1-null, mca2-null, and double mutants compared to the wild type (Figure 3). Elongation growth suppression was detected at a lower gravity level $(30 \mathrm{~g})$ in the MCA1- and $M C A 2$-overexpressing mutants (Figure 4), indicating that the $M C A$-overexpressing seedlings were more sensitive to hypergravity. These results support the hypothesis that MCAs are involved in the perception of gravity when Arabidopsis hypocotyls are subjected to hypergravity.

The suppression of hypocotyl elongation under $300 \mathrm{~g}$ and $500 \mathrm{~g}$ hypergravity conditions was similar between the mca1-null, mca2-null, and double mutants, although the suppression ratio was smaller than that of wild type; all were $45 \%-70 \%$ of the wild type (Figure 3 ). These results suggest that the functions of MCA1 and MCA2 overlap, and that other mechanosensitive channels are also involved in gravity signal perception. Possible candidates are MSL9 and MSL10, the Arabidopsis homologs of the bacterial mechanosensitive channel of small conductance (MscS), which has mechanosensitive channel activity in the plasma membrane of root cells [22]. The involvement of MSLs in the plant response to hypergravity is an interesting topic for future studies.

Elongation in Arabidopsis hypocotyls occurs in the upper 12 cells of the epidermal cell file, which is suppressed by hypergravity [14]. The results of growth of mca mutants (Figures 3 and 4) suggest that MCAs are involved in the perception of gravity in this elongation region. On the other hand, the expression levels of MCAs increased in all regions of the hypocotyls under hypergravity conditions, particularly in the basal region (Figures 1 and 2), which plays a role in supporting the whole plant body. Thus, MCAs may be involved in the gravity perception in all regions of the hypocotyls, and their roles in the supporting region might be especially important for plants.

Here, we analyzed changes in the expression of $M C A$ genes and the growth of mca mutants in hypergravity conditions. In general, shoot organs grow at half the rate of the $1 \mathrm{~g}$ control, even at $300 \mathrm{~g}$ [1]. The growth and cell wall parameters vary in proportion to the logarithm of the magnitude of gravity, and hypergravity at $300 \mathrm{~g}$ causes about half the changes observed in microgravity in space (ca $10^{-4} \mathrm{~g}$ ) [1]. Moreover, the effects of $300 \mathrm{~g}$ hypergravity on growth and the cell wall were restored when the plant materials were transferred to $1 \mathrm{~g}$ conditions, and were completely nullified by the presence of gadolinium ions, as mentioned above. These results indicate that hypergravity at $300 \mathrm{~g}$ is not an extraordinary stimulus for plants, and that the plant response to this magnitude of gravity stress is within the normal physiological limits. However, the role of MCAs in the perception of gravity signals at $1 g$ has yet to be determined. For this purpose, analyses under true microgravity conditions in space may be most effective as the control; a space experiment termed plant gravity sensing (PI: H. Tatsumi) is now underway. 


\section{Materials and Methods}

\subsection{Plant Materials and Growth Analysis}

The Columbia-0 ecotype of Arabidopsis and its transgenic lines mca1-null, mca2-null, mca1-null mca2-null, MCA1-overexpressing, MCA2-overexpressing, MCA1p::GUS, and MCA2p::GUS were used in this study. The details of these lines have been previously described $[15,16]$. Seeds were sown on $1 \%$ $(w / v)$ agar in a $50 \mathrm{ml}$ centrifuge tube, incubated at $4{ }^{\circ} \mathrm{C}$ in the dark for 4 days, and then exposed to light $\left(50 \mu \mathrm{mol} \mathrm{m} \mathrm{m}^{-2} \mathrm{~s}^{-1}\right.$, red: blue $\left.=2: 1\right)$ at $23{ }^{\circ} \mathrm{C}$ for 1 day to induce germination. After germination, the seeds were incubated at $25^{\circ} \mathrm{C}$ in the dark for 1 day. Hypergravity experiments were conducted as reported previously [23]. The seedlings were exposed to hypergravity at 30-500 $g$ (via centrifugation; H-28-F, Kokusan, Tokyo, Japan) at $25{ }^{\circ} \mathrm{C}$ for 1 day in the dark. After treatment, the lengths of the hypocotyls were measured using a scale. The suppression ratio was calculated as a ratio (percentage) of the difference between the elongation under $1 g$ and the hypergravity conditions to the $1 g$ value. Because the lengths of hypocotyls at $1 \mathrm{~g}$ were different from line to line, the elongation growth data were compared between the $1 g$ and hypergravity treatments in each line and analyzed with Student's $t$-test (upper and middle panels of Figures 3 and 4). The suppression ratio data were analyzed using the Tukey HSD test (lower panels of Figures 3 and 4).

\subsection{Analysis of MCA Gene Expression}

Seedlings were fixed in $90 \%$ acetone in the dark for $1 \mathrm{~h}$ and washed twice with $0.1 \mathrm{M}$ sodium phosphate buffer, $\mathrm{pH}$ 7.0. The seedlings were then incubated at $37^{\circ} \mathrm{C}$ for $20 \mathrm{~h}$ with X-Gluc buffer (0.5. $\mathrm{mg} \mathrm{mL}^{-1}$ 5-bromo-4-chloro-3-indolyl- $\beta$-D-glucuronic acid (BMS), $0.1 \mathrm{M}$ sodium phosphate buffer, $\mathrm{pH} 7.0,0.5 \mathrm{mM}$ potassium ferrocyanide, and $0.5 \mathrm{mM}$ potassium ferricyanide) after infiltration with a vacuum pump for $1 \mathrm{~h}$. The stained seedlings were fixed in a mixed solution (ethanol: acetic acid = 3:1) for $5 \mathrm{~h}$, washed stepwise with $70 \%, 50 \%$, and $20 \%$ ethanol for $10 \mathrm{~min}$ each, and cleared overnight at $4{ }^{\circ} \mathrm{C}$ with a cleaning solution (chloral hydrate: glycerol: water = 8:1:3). Quantification of the GUS-staining intensity of each region of the hypocotyl was performed using ImageJ, as reported previously [24]. The GUS-staining intensity data (Figures 1 and 2) were analyzed using Student's $t$-test.

\section{Conclusions}

MCA1 and MCA2 are at least partly involved in gravity perception when Arabidopsis hypocotyls are exposed to hypergravity. These genes may be responsible for gravity signal perception in the gravity resistance of plants. Future studies should examine the involvement of MCA proteins in plants' resistance to $1 \mathrm{~g}$ gravity by space experiments.

Author Contributions: T.H. (Takayuki Hoson) conceived the study; K.S., K.W., H.I. and T.H. (Takayuki Hoson) designed the project; T.H. (Takayuki Hattori), Y.O. and Y.N. conducted the experiments and analyzed the data; T.H. (Takayuki Hattori), K.S., K.W. and T.H. (Takayuki Hoson) wrote the manuscript; Y.O., Y.N., and H.I. confirmed the manuscript. All authors have read and agreed to the published version of the manuscript.

Funding: This study was supported in part by Grants-in-Aid for Scientific Research (KAKENHI No. 25514006) from the Japan Society for the Promotion of Science.

Acknowledgments: We thank our respective laboratory members for their discussion and advice regarding the present study.

Conflicts of Interest: The authors declare no conflicts of interest.

\section{References}

1. Hoson, T.; Soga, K. New aspects of gravity responses in plant cells. Int. Rev. Cytol. 2003, 229, $209-244$. [PubMed]

2. Hoson, T.; Saito, Y.; Soga, K.; Wakabayashi, K. Signal perception, transduction, and response in gravity resistance. Another graviresponse in plants. Adv. Space Res. 2005, 36, 1196-1202. [CrossRef] 
3. Hoson, T.; Wakabayashi, K. Role of the plant cell wall in gravity resistance. Phytochemistry 2015, 112, 84-90. [CrossRef] [PubMed]

4. Soga, K.; Wakabayashi, K.; Kamisaka, S.; Hoson, T. Hypergravity induces reorientation of cortical microtubules and modifies growth anisotropy in azuki bean epicotyls. Planta 2006, 224, 1485-1494. [CrossRef]

5. Wasteneys, G.O.; Galway, M.E. Remodeling the cytoskeleton for growth and form: An overview with some new views. Annu. Rev. Plant Biol. 2003, 54, 691-722. [CrossRef]

6. Baskin, T.I. Anisotropic expansion of the plant cell wall. Annu. Rev. Cell Dev. Biol. 2005, 21, $203-222$. [CrossRef]

7. Matsumoto, S.; Saito, Y.; Kumasaki, S.; Soga, K.; Wakabayashi, K.; Hoson, T. Up-regulation of tubulin genes and roles of microtubules in hypergravity-induced growth modifications in Arabidopsis hypocotyls. Adv. Space Res. 2007, 39, 1176-1181. [CrossRef]

8. Soga, K.; Kotake, T.; Wakabayashi, K.; Kamisaka, S.; Hoson, T. Transient increase in the transcript levels of $\gamma$-tubulin complex genes during reorientation of cortical microtubules by gravity in azuki bean (Vigna angularis) epicotyls. J. Plant Res. 2008, 121, 493-498. [CrossRef]

9. Soga, K.; Kotake, T.; Wakabayashi, K.; Kamisaka, S.; Hoson, T. The transcript level of katanin gene is increased transiently in response to changes in gravitational conditions in azuki bean epicotyls. Biol. Sci. Space 2009, 23, 23-28. [CrossRef]

10. Ding, J.P.; Pickard, B.G. Mechanosensory calcium-selective cation channels in epidermal cells. Plant J. 1993, 3, 83-110. [CrossRef]

11. Fasano, J.M.; Massa, G.D.; Gilroy, S. Ionic signaling in plant responses to gravity and touch. J. Plant Growth Regul. 2002, 21, 71-88. [CrossRef] [PubMed]

12. Soga, K.; Wakabayashi, K.; Kamisaka, S.; Hoson, T. Graviperception ingrowth inhibition of plant shoots under hypergravity conditions producedby centrifugation is independent of that in gravitropism and may involve mechanoreceptors. Planta 2004, 218, 1054-1061. [PubMed]

13. Soga, K.; Wakabayashi, K.; Kamisaka, S.; Hoson, T. Hypergravity inhibits elongation growth of azuki bean epicotyls independently of the direction of stimuli. Adv. Space Res. 2005, 36, 1269-1276. [CrossRef]

14. Matsumoto, S.; Kumasaki, S.; Soga, K.; Wakabayashi, K.; Hashimoto, T.; Hoson, T. Gravity-induced modifications to development in hypocotyls of Arabidopsis tubulin mutants. Plant Physiol. 2010, 152, 918-926. [CrossRef] [PubMed]

15. Nakagawa, Y.; Katagiri, T.; Shinozaki, K.; Qi, Z.; Tatsumi, H.; Furuichi, T.; Kishigami, A.; Sokabe, M.; Kojima, I.; Sato, S.; et al. Arabidopsis plasma membrane protein crucial for $\mathrm{Ca}^{2+}$ influx and touch sensing in roots. Proc. Natl. Acad. Sci. USA 2007, 104, 3639-3644. [CrossRef]

16. Yamanaka, T.; Nakagawa, Y.; Mori, K.; Nakano, M.; Imamura, T.; Kataoka, H.; Terashima, A.; Iida, K.; Kojima, I.; Katagiri, T.; et al. MCA1 and MCA2 that mediate $\mathrm{Ca}^{2+}$ uptake have distinct and overlapping roles in Arabidopsis. Plant Physiol. 2010, 152, 1284-1296. [CrossRef]

17. Kamano, S.; Kume, S.; Iida, K.; Lei, K.J.; Nakano, M.; Nakayama, Y.; Iida, H. Transmembrane Topologies of $\mathrm{Ca}^{2+}$-permeable Mechanosensitive Channels MCA1 and MCA2 in Arabidopsis thaliana. J. Biol. Chem. 2015, 290, 30901-30909. [CrossRef]

18. Kurusu, T.; Yamanaka, T.; Nakano, M.; Takiguchi, A.; Ogasawara, Y.; Hayashi, T.; Iida, K.; Hanamata, S.; Shinozaki, K.; Iida, H.; et al. Involvement of the putative $\mathrm{Ca}^{2+}$-permeable mechanosensitive channels, NtMCA1 and NtMCA2, in $\mathrm{Ca}^{2+}$ uptake, $\mathrm{Ca}^{2+}$-dependent cell proliferation and mechanical stress-induced gene expression in tobacco (Nicotiana tabacum) BY-2 cells. J. Plant Res. 2012, 125, 555-568. [CrossRef]

19. Furuichi, T.; Iida, H.; Sokabe, M.; Tatsumi, H. Expression of Arabidopsis MCA1 enhanced mechanosensitive channel activity in the Xenopus laevis oocyte plasma membrane. Plant Signal. Behav. 2012, 7, 1022-1026. [CrossRef]

20. Mori, K.; Renhu, N.; Naito, M.; Nakamura, A.; Shiba, H.; Yamamoto, T.; Suzaki, T.; Iida, H.; Miura, K. $\mathrm{Ca}^{2+}$-permeable mechanosensitive channels MCA1 and MCA2 mediate cold-induced cytosolic $\mathrm{Ca}^{2+}$ increase and cold tolerance in Arabidopsis. Sci. Rep. 2018, 8, 550. [CrossRef]

21. Hoson, T. Interaction of gravity with other environmental factors in growth and development: An introduction. Adv. Space Res. 1999, 23, 1971-1974. [CrossRef]

22. Haswell, E.S.; Peyronnet, R.; Barbier-Brygoo, H.; Meyerowitz, E.M.; Frachisse, J.M. Two MscS homologs provide mechanosensitive channel activities in the Arabidopsis root. Curr. Biol. 2008, 18, 730-734. [CrossRef] [PubMed] 
23. Soga, K.; Yano, S.; Matsumoto, S.; Hoson, T. Plant Gravitropism. Methods in Molecular Biology; Blancaflor, E., Ed.; Humana Press: New York, NY, USA, 2015; Volume 1309, pp. 307-319.

24. Béziat, C.; Kleine-Vehn, J.; Feraru, E. Histochemical staining of $\beta$-glucuronidase and its spatial quantification. In Plant Hormones. Methods in Molecular Biology; Kleine-Vehn, J., Sauer, M., Eds.; Humana Press: New York, NY, USA, 2017; Volume 1497, pp. 73-80. 